\title{
Investigating the complexity of computer-supported collaborative learning in action
}

\section{U. Cress ${ }^{1} \cdot$ C. P. Rosé ${ }^{2} \cdot$ N. Law ${ }^{3} \cdot$ S. Ludvigsen ${ }^{4}$}

Published online: 22 August 2019

(C) International Society of the Learning Sciences, Inc. 2019

\section{Introduction}

One of the most notable developments in computer-supported collaborative learning (CSCL) over the years has dealt with the scale and scope of the research. Early CSCL research typically investigated scenarios that were quite restricted (e.g., by doing controlled laboratory experiments on non-representative student samples, focusing on learning outcomes rather than learning processes, or by analyzing snippets of interaction). However, as our field matures, research increasingly addresses the complexity of real CSCL settings as they unfold. As a consequence, much contemporary CSCL research not only focuses on the core of CSCL (technologically mediated peer-learning interactions) but also on how CSCL interactions may develop within larger social systems and practices, taking account of the contextual constraints and affordances. The papers in the current issue of the International Journal of ComputerSupported Collaborative Learning all provide excellent examples of how CSCL's core processes are affected by a combination of intentional design elements and properties of existing contexts.

When CSCL is an integral part of regular classroom practice, the context of the collaborative interaction is largely defined by teachers who orchestrate, monitor, and guide CSCL activities among peer learners (Matuk and Linn 2018). In other CSCL contexts, embedded

\section{S. Ludvigsen}

s.r.ludvigsen@iped.uio.no

U. Cress

u.cress@iwm-tuebingen.de

C. P. Rosé

cprose@cs.cmu.edu

N. Law

nlaw@hku.hk

1 Leibniz-Institut für Wissensmedien (Knowledge Media Research Center), Tübingen, Germany

2 Carnegie Mellon University Language Technologies Institute and HCI Institute, Pittsburgh, PA, USA

3 University of Hong Kong, Hong Kong, China

4 University of Oslo, Oslo, Norway 
designs bring learners into contact with practitioners from other professional fields or simulate authentic learning environments in the classroom.

A taxonomy for conceptualizing authenticity is the focus of the first paper (Hod \& Sagy). The second paper in this issue (van Heijst, de Jong, van Aalst, de Hoog, \& Kirschner) investigates how the dynamics of CSCL discourse may be affected by the socio-cognitive openness of the context. In the third paper, Slakmon and Schwarz raise questions about how to balance social, cognitive, and emotional aspects in hot-topic discussions. They show that emotions are often neglected both conceptually and empirically. They also demonstrate how emotions can be accounted for and how we can design CSCL environments that involve emotions in hot-topic discussions.

The final two contributions are responses to the ongoing discussions based on the Wise and Schwarz (2017) squib which raised eight provocations for the field. The squib contribution from Borge and Mercier reflects on the idea that a proper analysis of the contextual richness of CSCL activities requires a micro-ecological approach that might go beyond standard theoretical conceptualizations. In the other squib, Tchounikine argues that we need to take account of the learner's agency and understand how emancipatory agency can be realized in CSCL settings. The authors question many established assumptions in the CSCL field. Both squibs should provoke the CSCL community to continue the discussion.

\section{Authentic learning contexts for CSCL: a taxonomy}

Socio-cultural theories of learning have made important contributions to our understanding of contextual factors. A central tenet of these approaches is that learning is a process of enculturation; through participation in the activities of a community of practice, learners acquire the norms, values, and skills that shape the core identity of the community (Brown et al. 1989; White 2018). Socio-cultural theories have therefore posited that enculturation requires students to get into contact with the practices of different professional cultures, a claim that is generally subsumed under the umbrella term "authentic learning environments" (Edelson and Reiser 2006). Many empirical studies have provided excellent examples of how the authenticity of learning environments can be established or increased. However, due to the vast scope of contexts found in studies of classroom practice, it is difficult to conduct systematic comparisons of authenticity in empirical studies.

In order to address this difficulty, the paper by Hod and Sagy offers a taxonomy and conceptualization to categorize and compare different authentic learning scenarios. This conceptualization builds on a basic distinction between simulation environments and hybrid environments. In a simulated environment within a classroom context, attempts are made to match or approximate a learning environment to an authentic culture. In a hybrid environment, students interact with practitioners of a professional culture, and these interactions at least partially take place in the professional setting itself.

Hod and Sagy reviewed a corpus of more than 40 scientific papers that describe authentic learning environments, focusing on 23 articles that provide extensive information about authentic design. The top level of their classification of the learning environments comprised two categories: simulation or hybrid. For each of these two broad categories, Hod and Sagy propose a list of five descriptors to characterize the different contexts and scenarios: type of participants, the setting, the temporal dimension of collaboration (timeframe), the kind of computer support used, and the if the collaboration was meaningful. The authors have also 
developed a specific type of visualization that displays the relations between participants, settings, and cultures. This helps to identify commonalities and differences between authentic learning environments.

Based on their review the authors identified a baseline simulation approach, a baseline hybrid approach, and characteristic variations of these baseline types. By matching the two different basic approaches and their variants to the five dimensions, Hod and Sagy identify meaningful patterns. For instance, simulation and hybrid approaches do not necessarily differ with regard to participants or the extent they experience the collaboration as meaningful.

Many empirical papers have provided best-practice examples of how learning environments can be designed. However, as many of these papers present case studies, they are difficult to compare. The taxonomy by Hod and Sagy provides a framework for classifying authentic learning environments that can be used to assess the existing fieldwork and to guide future research.

\section{Cognitive and social openness: a new construct for CSCL?}

While the other contributions in this issue address CSCL contextual factors on a meso level (e.g., in orchestrating classroom activities), the paper by van Heijst et al. examines the contextual factors of the core CSCL processes themselves.

Knowledge-building through the mutual refinement of ideas and explanations is generally regarded as the key process in CSCL activities. Yet, while knowledge building is at the heart of CSCL, many empirical studies have shown that the actual levels of knowledge-building reached are not very high. Van Heijst et al. discuss how students sometimes do not build upon the prior work of their peers and need scaffolding to do so (Wang et al. 2017). This led the authors to develop a new explanatory construct for CSCL, which they call socio-cognitive openness. Based on the focal constructs developed by various CSCL scholars, van Heijst et al. distinguish between two dimensions of openness (cognitive and social) that can be further divided into four knowledge-building discourse acts (knowledge-building, the expression of uncertainty, community orientation, the expression of self) and into eight different components. Van Heijst et al. have empirically tested the framework of socio-cognitive openness by careful analysis and coding of Knowledge Forum contributions. The subjects were two mixed cohorts of students and teachers that participated in courses with the theme of learning and innovation. After validating the two-factor structure of the socio-cognitive openness construct, van Heijen et al. investigated the frequency of the eight components in student's discourse and the likelihood that the expression of a particular component led to follow-up.

Results indicated that three of the eight components were present in the large majority of Knowledge Forum notes (connecting knowledge, transactivity, authority). Interestingly, two of these components of openness (transactivity, authority) were associated with decreased rather than increased likelihood of follow-up. However, when participants were connecting knowledge, justifying knowledge (which they rarely did), and inviting responses (which they also rarely did), their notes were more likely to receive follow-up. In other words, whereas components of cognitive openness seemed to invite further knowledge-building, expressing social openness actually had slightly detrimental effects on follow-up.

The notion of socio-cognitive openness offers some promising potential to advance our understanding of the contextual factors that influence knowledge-building activities. The expression of openness is a great candidate in describing the glue that creates knowledge- 
building. Further conceptual and empirical research could try to combine socio-cognitive openness with related factors in social sciences, such as trust (Balliet and Van Lange 2013) or social capital (Coleman 1988).

\section{Talk, cognition, and emotions in CSCL settings}

Talk, artefacts, and social interaction constitute the foundation for supporting CSCL participants. Particular CSCL designs can support, facilitate, and structure how cognition and emotions play out in collaborative efforts. In CSCL (as in many other fields of knowledge), emotions have not been given adequate attention. One exception is the paper by Polo et al. (2016) published in a previous edition of this journal; it analyzes emotion as part of social and cognitive processes in argumentation.

In "Deliberate Emotional Talk," the paper by Slakmon and Schwartz, emotions are treated as central aspects when students engage in dialogue on hot and controversial topics. The perspectives that inform the foundation of the paper are very rich. The authors draw on ideas and research from political education, dialogical theory, cognitive development, and CSCL studies. The review shows that emotions are seldom explicitly analyzed in contexts such as conversations. The authors apply a critical review to the classical contributions of Piaget as well as more recent dialogical approaches (Mercer and Dawes 2014; Perret-Clermont 1980). The authors emphasize that in the conceptualization of conversations, social aspects and the balance between cognition and emotions need to be considered.

In this study, the digital environment in which the students engaged in conversation is called the "Hot Discussions Platform (HDP)." This platform affords both written and oral contributions. The oral contributions take place in small groups. The design also includes a reflection mode in which a moderator can freeze the discussion or make select aspects of the discourse available for participants. The selected aspects can be used as resources for a new layer of discourse. The subjects in the study were 25 teachers enrolled in a course on professional development. The participants were assigned to different group configurations during the course. They were given open-ended tasks that were potentially hot and controversial.

The findings are interesting and point to new directions for CSCL research. Given that digital environments such as hot-topic discussion platforms are viewed as lived spaces for todays' generation of students, they will become increasingly important in educational practices. The implication for CSCL is that we need to design more refined and nuanced tools and environments connected to large infrastructure that can provide data for improvement. Such improvement also needs to be based on studies of social practices, such as that of Slakmon and Schwarz in this edition.

\section{New squibs: two new contribution to the provocations - the conversation continues}

The ijCSCL paper by Wise and Schwarz (2017) was poignant and provocative, and it is encouraging that it continues to garner "squib" responses from the CSCL community. In this issue, we publish two squibs, one by Marcela Borge and Emma Mercier and the other by Pierre Tchounikine. 
Borge and Mercier provide a brief overview of the four overarching learning theories within CSCL (information-processing perspective, constructivism, socio-constructivist/sociocultural approaches, group cognition), arguing that each of these approaches has developed theoretical lenses that are too narrow to capture the true contextual richness of CSCL in action. As an alternative to the four perspectives, Borge and Mercier propose a micro-ecological approach that cuts across multiple levels of analysis (individual, group, community) and analyzes how each of these levels influences and is influenced by the other levels.

Borge and Mercier argue that, for instance, simply analyzing episodes on one level would leave many events unexplained (e.g., why the facilitators came up with different orchestration moves, why one student destroyed the Minecraft world). A full picture could only emerge if one also looks at activities at the community/group level (e.g., it was one particular group that noticed the server failure) and the individual level (e.g., it was one student who tried to individually solve the technical problem before facilitators turned that effort into a collaborative endeavor). It remains to be seen if such a micro-ecological approach could offer a new analytical lens, since the socio-cultural perspective has combined various levels of analysis for many years (Steier et al. 2019; Solli et al. 2018; White 2018). The approach developed by Cress and colleagues has also worked to connect levels of analysis, such as cognition and social systems (Cress and Kimmerle 2008).

Whether a micro-ecological approach could bridge the gaps between different epistemologies in CSCL and provide a viable solution for the commensurability in Wise and Schwarz's (2017) provocations is an interesting problem. In any case, the squib by Borge and Mercier is an important reminder that many of us tend to see the field of CSCL through a particular theoretical lens.

In the squib by Tchounikine, the author raises questions about how CSCL treats the learner's agency. He argues that CSCL settings are often predefined for learners. Participants are expected to use specific features of a setting or environment but not to choose digital tools (types of software) of their own selection as part of their learning. Tchounikine emphasizes that we should start understanding participants' actions and activities from an emancipatory perspective. This implies that participants should be given the opportunity to select, change, and combine the technologies that they think would be most beneficial for their learning trajectories. Such trajectories would not be possible without a deep appropriation of a set of different digital tools.

Do Tchounikine's arguments exclude CSCL designs? He argues not, but design should be envisioned from the perspective of participants' agency. The use of digital tools and environments can bring us down unexpected paths, which means that what can be learned cannot be fully defined in advance. Learning can become an expansive activity beyond what is given in predefined tasks.

Empowering participants through emancipation might imply different forms of feedback. In CSCL, learning analytics are an emerging and important topic. Analytics for supporting collaborative learning have a long history in CSCL, and many core CSCL concepts should be seen as part of supporting participants in their engagement. Tchounikine views analytics from the perspective of how it can emancipate the learner's agency. He argues that feedback should be viewed as a means for raising awareness and recommendations that the learner must choose to act on. From an editorial perspective, one can ask is Tchounikine's line of argument realistic or romantic? We hope our readers can examine and engage with the problems that are emphasized in this squib. 


\section{Conclusion}

The mutual refinement of ideas through interaction, arguably constitutes the core process of CSCL. However, the contributions in this issue of the IJCSCL nicely illustrate how this core process is embedded within multiple contextual layers, which influence the conversations and interactions in multiple ways, each of which needs to be analyzed, understood, and ultimately integrated into the orchestration and facilitation of CSCL activities.

\section{References}

Balliet, D., \& Van Lange, P. A. M. (2013). Trust, conflict, and cooperation: A meta-analysis. Psychological Bulletin, 139, 1090-1112.

Brown, J. S., Collins, A., Duguid, P., \& Brown, S. (1989). Situated cognition and the culture of learning. Educational Researcher, 18(1), 32-42.

Coleman, J. S. (1988). Social capital in the creation of human capital. American Journal of Sociology, 94, 95-120.

Cress, U., \& Kimmerle, J. (2008). A systemic and cognitive view on collaborative knowledge building with wikis. International Journal of Computer-Supported Collaborative Learning, 3(2), 105-122.

Edelson, D., \& Reiser, B. (2006). Making authentic practices accessible to learning: Design challenges and strategies. In R. K. Sawyer (Ed.), The Cambridge handbook of the learning sciences (pp. 335-354). New York: Cambridge University Press.

Matuk, C., \& Linn, M. C. (2018). Why and how do middle school students exchange ideas during science inquiry? International Journal of Computer-Supported Collaborative Learning, 13(3), 263-299. https://doi.org/10.1007/s11412-018-9282-1.

Mercer, N., \& Dawes, L. (2014). The study of talk between teachers and students, from the 1970s until the 2010s. Oxford Review of Education, 40(4), 430-445.

Perret-Clermont, A. N. (1980). Social interaction and cognitive development in children. London: Academic.

Polo, C., Lund, K., Plantin, C., \& Niccolai, G. P. (2016). Group emotions: The social and cognitive functions of emotions in argumentation. International Journal of Computer-Supported Collaborative Learning, 11(2), $123-156$.

Solli, A., Mäkitalo, Å., \& Hillman, T. (2018). Rendering controversial socioscientific issues legible through digital mapping tools. International Journal of Computer-Supported Collaborative Learning, 13, 391-418.

Steier, R., Kersting, M., \& Silseth, K. (2019). Imagining with improvised representations in CSCL environments. International Journal of Computer-Supported Collaborative Learning, 14(1), 109-136.

Wang, X., Kollar, I., Stegmann, K., \& Intern, J. (2017). Adaptable scripting to foster regulation processes and skills in computer-supported collaborative learning. International Journal of Computer-Supported Collaborative Learning, 12(2), 153-172. https://doi.org/10.1007/s11412-017-9254-x.

White, T. (2018). Connecting levels of activity with classroom network technology. International Journal of Computer-Supported Collaborative Learning, 13(1), 93-122. https://doi.org/10.1007/s11412-018-9272-3.

Wise, A. F., \& Schwarz, B. B. (2017). Visions of CSCL: Eight provocations for the future of the field. International Journal of Computer-Supported Collaborative Learning, 12(4), 423-467.

Publisher's note Springer Nature remains neutral with regard to jurisdictional claims in published maps and institutional affiliations. 